\title{
Deciphering the Enigma of Wave-Particle Duality
}

\author{
Mani L. Bhaumik \\ Department of Physics and Astronomy, University of California, Los Angeles, USA \\ E-mail:bhaumik@physics.ucla.edu
}

Editors: Zvi Bern \& Danko Georgiev

Article history: Submitted on October 17, 2016; Accepted on November 28, 2016; Published on December 7, 2016.

\begin{abstract}
A satisfactory explanation of the confounding wave-particle duality of matter is presented in terms of the reality of the wave nature of a particle. In this view, a quantum particle is an objectively real wave packet consisting of irregular disturbances of underlying quantum fields. It travels holistically as a unit and thereby acts as a particle. Only the totality of the entire wave packet at any instance embodies all the conserved quantities, for example the energy-momentum, rest mass, and charge of the particle, and as such must be acquired all at once during detection. On this basis, many of the bizarre behaviors observed in the quantum domain, such as wave function collapse, the limitation of prediction to only a probability rather than an actuality, the apparent simultaneous existence of a particle in more than one place, and the inherent uncertainty can be reasonably comprehended. The necessity of acquiring the wave function in its entirety for detection, as evinced by the appearance of collapse of the wave function, supports the paradigm of reality of the wave function described here.

Quanta 2016; 5: 93-100.
\end{abstract}

(c) This is an open access article distributed under the terms of the Creative Commons Attribution License CC-BY-3.0, which permits unrestricted use, distribution, and reproduction in any medium, provided the original author and source are credited.

\section{Introduction}

Ever since the advent of the wildly successful quantum theory nearly a century ago, its antecedent, wherein the wave aspect is always associated with a particle has been a source of great mystery to professional scientists and to the public as well. Louis de Broglie, who offered the hypothesis, considered the wave to be a fictitious pilot wave that guides a particle. Almost a quarter of a century later, David Bohm came up with the notion of a quantum potential instead, providing the quantum wave that guides the classical particle. He never expounded, however, on the source of energy of the quantum potential. Nonetheless, due to other appealing aspects of Bohm's theory such as the introduction of the concept of nonlocality, a small segment of the scientific community still seem to grant it credence.

Niels Bohr, the principal architect of the Copenhagen interpretation, was content to accept the duality paradox as an elementary aspect of the natural world. In his view, a quantum object will exhibit its wave aspect at some times and its particle nature at others, depending on the circumstances. He considered such duality an essential feature of complementarity, which he presumed to be an inherent property of nature.

During all this time, buoyed by the phenomenal success and the superb predictive power of the quantum theory, most practicing physicists avoided the conundrum by treating the wave function as merely a fictitious mathematical construct to be used for the algorithm called quantum theory. 
This caused the pioneering proponent of the quantum theory, Albert Einstein, great consternation. He posited, "This double nature of radiation (and of material corpuscles) ... has been interpreted by quantum-mechanics in an ingenious and amazingly successful fashion. This interpretation ... appears to me as only a temporary way out ..." [1, p. 51]

"At the heart of the problem," Einstein said of quantum mechanics, "is not so much the question of causality but the question of realism." [2, p. 460] Einstein's insistence on the reality of the wave function, however, remained conspicuously dormant until recently, when it became the subject of lively discussion in the form of $\psi$-ontic versus $\psi$-epistemic debate. A comprehensive review has been provided by Leifer [3]. It would appear that $\psi$-ontic theories advanced by Colbeck and Renner [4] and Pusey, Barrett, and Rudolph [5], which advocate the objective reality of the wave function, are gaining more traction. Their presentation, however, is based primarily on an information-theoretic viewpoint and does not take into account physical aspects such as the conservation of energy or momentum.

In this work, we provide a paradigm of the reality of the wave function based on energy-momentum considerations that cogently explicates the enigma of the wave-particle duality, an enigma which is inseparably connected with several other conundrums of quantum physics, such as, Heisenberg's uncertainty principle, the Born rule of probability, collapse of the wave function, and the apparent simultaneous existence of a particle in more than one place. The concept of the objective reality presented here solely in terms of physical parameters is more explicit, albeit for a single particle, and ought to harmonize with the information-theoretic approach.

The topic of wave-particle duality itself has a fascinating history. Notably, it was Einstein himself who fostered this innovative notion by advocating the real existence of quanta of radiation or photons. Previously Maxwell and others had convincingly established the wave nature of electromagnetic radiation. An abundance of experiments on the interference, diffraction, and scattering of light had substantiated it beyond any reasonable doubt. Thus, it was greeted with utter shock and disbelief when Einstein argued in 1905 that under certain circumstances light behaves not as continuous waves but as discontinuous, individual particles [6,7]. These particles, or "light quanta," each carried a "quantum," or fixed amount, of energy.

In the face of the almost unanimous opposition of his peers, Einstein remained perhaps the principal champion of the wave-particle duality of radiation for almost two decades, until he was finally vindicated by the spectacular observation of the particle aspect in the Compton effect in 1923. The following year, de Broglie extended the idea of wave-particle duality to matter particles with enthusiastic support from Einstein.

Soon the evidence for the matter wave came along with the apparent accidental discovery of electron waves by Clinton Davisson and Lester Germer in observing a diffraction pattern in the beam of electrons scattered by nickel crystal [8]. Shortly before that, George Paget Thomson and Alexander Reid also provided some evidence of matter waves by detecting a diffraction pattern when electrons passed through a very thin metallic foil [9]. Both Davisson and Thompson shared the 1937 Nobel Prize in Physics for their startling discovery, ushering the age of quantum physics in earnest.

In the meantime Erwin Schrödinger, inspired by de Broglie and Einstein, formulated the wave mechanics of quantum physics, replacing the particle in classical mechanics with a wave function [10].

\section{Reality of the wave function}

In previous work [11], we presented a credible argument in favor of the existence of an objective reality behind the wave function at the core of quantum physics. A synopsis is presented here. The ontology of the wave function advocated in this paper is primarily grounded on the incontrovertible physical evidence that all electrons in the universe are exactly identical.

The answer to the long standing puzzle of why all electrons in all respects are universally identical, a feature eventually found to be true as well of all the other fundamental particles, was finally provided by the quantum field theory of the Standard Model of particle physics constructed by combining Einstein's special theory of relativity with quantum physics, a science which has evolved from his own pioneering contributions.

Nobel Laureate in Physics, Steven Weinberg [12], declares that quantum field theory is an unavoidable consequence of the reconciliation of quantum mechanics with special relativity. It has successfully explained almost all experimental observations in particle physics and correctly predicted a wide assortment of phenomena with impeccable precision. By way of many experiments over the years, the quantum field theory of Standard Model has become recognized as a well-established theory of physics. Another Nobel Laureate David Gross asserts that all the pieces of the puzzle of the Standard Model of particles physics fit beautifully in quantum field theory of the Standard Model such that there are no more pieces of the puzzle left to fit (private communication). Yet another Physics Nobel Laureate, Franck Wilczek underscores, "the standard model is very successful in de- 
scribing reality-the reality we find ourselves inhabiting" [13, p. 96]. Expressions of such confidence encourage us to anchor our reliance on it.

Although one might argue that the Standard Model accurately describes the phenomena within its domain, it is still incomplete as it does not include gravity, dark matter, dark energy, and other phenomena. However, because of its astonishing success so far, whatever deeper physics may be necessary for its completion would very likely extend its scope without repudiating its current depiction of fundamental reality.

According to quantum field theory, the fundamental particles which underpin our daily physical reality are only secondary. Each fundamental particle, whether it is a boson or a fermion, originates from its corresponding underlying quantum field [14-17]. The particles are excitations of quantum fields possessing propagating states of discrete energies, and it is these fields which constitute the primary reality. For example, a photon is a quantum of excitation of the photon field (aka electromagnetic field), an electron is a quantum of the electron quantum field, and a quark is a quantum of the quark quantum field, and so on for all the fundamental particles of the universe.

By far, the most phenomenal step forward made by quantum field theory lies in the stunning prediction that the primary ingredient of everything in this universe is present in each element of spacetime $(x, y, z, t)$ of this immensely vast universe [13, p. 74]. These ingredients are the underlying quantum fields. We also realize that the quantum fields are alive with quantum activity. These activities have the unique property of being completely spontaneous and utterly unpredictable as to exactly when a particular event will occur [13, p. 74]. Furthermore, some of the quantum fluctuations occur at mind-boggling speeds with a typical time period of $10^{-21}$ seconds or less. In spite of these wild infinitely dynamic, fluctuations, the quantum fields have remained immutable, as evinced by their Lorentz invariance, essentially since the beginning and throughout the entire visible universe.

\section{Quantum particle in motion}

As elucidated above, an electron represents a propagating discrete quantum of the underlying electron field. In other words, an electron is a quantized wave (or a ripple) of the electron quantum field, acting as a particle because of its well-defined energy, momentum, and rest mass, which are conserved fundamentals of the electron. However, even a single electron, in its reference frame, is never alone. It is unavoidably subjected to the perpetual fluctuations of the quantum fields.
When an electron is created instantaneously from the electron quantum field, its position would be indefinite since a regular ripple with a very well defined energy and momentum is represented by a non-localized periodic function. However, the moment the electron comes into existence, quantum fluctuations facilitate its interaction with all the other quantum fields. For example, the presence of the electron creates a disturbance in the electromagnetic or the photon quantum field. Assisted by a fleeting quantum fluctuation, the disturbance in the photon field can momentarily appear as what is commonly known as a spontaneously emitted virtual photon. It is these interactions that allow the particle's position to be localized.

The quantum fluctuations continually and prodigiously create virtual electron-positron pairs in a volume surrounding the electron. "Each pair passes away soon after it comes into being, but new pairs are consistently boiling up to establish an equilibrium distribution" [18, p. 404]. Even though each pair has only a fleeting existence, on average there are a significant enough number of these pairs to generate a remarkably sizable screening of the bare charge of the electron.

Likewise, though any individual disturbances in the fields or the virtual particles due to quantum fluctuations have an ephemeral existence, there ought to be an equilibrium distribution of such disturbances present at any particular time and affecting other aspects of the electron. The effect of these disturbances is very well established in phenomena such as the Lamb shift and the anomalous gyromagnetic factor of the electron's spin.

The electron's spin $g$-factor has been measured to a precision of better than one part in trillion, compared to the theoretically calculated value that includes quantum electrodynamics diagrams up to four loops [19]. Therefore, it would be reasonable to assume that the average number of disturbances of all quantum fields present at any particular time will be strikingly stable in spite of their fleeting in and out of existence.

Recall again that an electron is a quantized ripple of the electron quantum field, acting as a particle because it travels with its conserved quantities always maintained holistically as a unit. However, due to interactions of the particle with all the other quantum fields, substantially equivalent to those involved in the Lamb shift and the observed spin $g$-factor, the ripple in fact becomes very highly distorted immediately after its creation since the quantum fluctuations causing the interactions of the quantum fields have a typical time period of $10^{-21}$ seconds. Consequently, it ceases to be a ripple of single frequency and becomes a highly deformed localized travelling pulse.

It is well known that such a pulse, no matter how deformed, can be expressed by a Fourier integral with 
weighted linear combinations of simple periodic wave forms like trigonometric functions, briefly mentioned by the author in an earlier work [20]. The result would be a wave packet or a wave function that represents a fundamental objective reality of the universe. Such a wave function would be smooth and continuously differentiable, especially using complex numbers in the weighted amplitude coefficients. The wave function $\psi(x)$ will be given by the Fourier integral,

$$
\psi(x)=\frac{1}{\sqrt{2 \pi}} \int_{-\infty}^{+\infty} \tilde{\psi}(k) e^{\imath k x} d k
$$

where $\tilde{\psi}(k)$ is a continuous function that determines the amount of each wave number component $k=2 \pi / \lambda$ that gets added to the combination.

From Fourier analysis, we also know that the spatial wave function $\psi(x)$ and the wave number function $\tilde{\psi}(k)$ are a Fourier transform pair. Therefore, we can find $\tilde{\psi}(k)$ through the Fourier transform of $\psi(x)$ :

$$
\tilde{\psi}(k)=\frac{1}{\sqrt{2 \pi}} \int_{-\infty}^{+\infty} \psi(x) e^{-l k x} d x .
$$

Thus, the Fourier transform relationship between $\psi(x)$ and $\tilde{\psi}(k)$, where $x$ and $k$ are known as conjugate variables, can help us determine the frequency or the wave number content of any spatial wave packet function.

\section{Time evolution of the wave packet}

In order to determine the time evolution of the wave packet function, we need to incorporate the time term to the spatial function. Accordingly,

$$
\psi(x, t)=\frac{1}{\sqrt{2 \pi}} \int_{-\infty}^{+\infty} \tilde{\psi}(k) e^{l(k x-\omega(k) t)} d k
$$

We introduced $\omega(k)$ since the angular frequency will be quite often a function of the wave number $k$. The wave packet function has a dominant central wave number $k_{0}$ and a range of additional wave numbers on either side that combines to provide the necessary localization of the packet. If we write the wave-packet function as

$$
W(x)=\frac{1}{\sqrt{2 \pi}} \int_{-\infty}^{+\infty} \tilde{\psi}(k) d k,
$$

the wave function becomes

$$
\psi(x, t)=W(x) e^{l(k x-\omega(k) t)} d k .
$$

The dominant wave number $k_{0}$ corresponds to the dominant angular frequency $\omega_{0}=2 \pi v_{0}=\frac{E_{0}}{\hbar}$ using the Planck relation $E=h v$.
The kinematics of the wave packet will conform to the relativistic energy relation,

$$
E^{2}=p^{2} c^{2}+m_{0}^{2} c^{4} .
$$

Or equivalently, in terms of the Plank-Einstein formula

$$
\begin{aligned}
E & =h v=m c^{2}, \\
h^{2} v^{2} & =h^{2} v_{0}^{2}+p^{2} c^{2} .
\end{aligned}
$$

Before the electro-weak symmetry breaking about a trillionth of a second after the Big Bang and the attendant manifestation of the Higgs field, all the wave packets representing the various particles having no mass were speeding along with the velocity of light $c$, since the group velocity of the wave-packet

$$
v_{g}=\frac{\partial \omega}{\partial k}=\frac{\partial(k c)}{\partial k}=c .
$$

A sweeping change occurred in the kinematics of the wave packets after their interaction with the Higgs field, when a wave packet is considered to have acquired a mass, more specifically the rest mass $m_{0}$, thereby reducing its translational motion [21, p. 368]. In other words, by interacting with the Higgs field, the wave packet, paraphrasing Einstein, has acquired inertia proportional to its energy content [22,23]. By this process, different wave packets representing different particles all acquire their rest masses, which is a measure of the strength of their coupling with the Higgs field. However, some particles like photons and gluons remain massless since they do not interact with the Higgs condensate. Consequently, equation (3) will still be valid for their kinematics, since the wave number in the phase factor is the same as that of the wave packet. But for a massive particle, some modification is needed to deal with their kinematics, as the wave number in the phase factor will be different than the wave number of the energy carrying wave packet.

Without any kinetic energy, a wave packet with the intrinsic energy $h v_{0}$ would become a standing wave packet corresponding to rest mass energy $m_{0} c^{2}$. The essential feature here is to recognize that even though the particle is at rest, its rest mass energy is not. It is manifest in the vibrations of a standing wave packet obeying the equation [24]

$$
\psi(x, t)=W(x) e^{-l E_{0} t / \hbar} .
$$

For an anti-particle, the negative sign in the exponent is replaced by a plus.

Since the temperature and energy of the universe following the manifestation of the Higgs field were still very high compared to the rest mass energy, the kinematics of the wave packet (particle) obeyed the relativistic energymomentum equation (6). However, the mean free path of 
any particle was rather small because of the rapid rate of pair production and annihilation inside the predominantly high energy photon gas.

Eventually, when the universe was about a few seconds old, it cooled down sufficiently below the threshold of all pair productions and nearly one in ten billion particles survived over the antiparticles as a result of asymmetry in bariogenesis during the early universe. The electrons were the last to escape pair production and annihilation in the primordial soup. Ultimately, the wave packets of matter particles combined to form atoms and other forms of matter.

\section{Modulation of the wave packet}

As described above, the holistic wave packet of a particle like an electron goes back to the very beginning of its creation. When an electron is at complete rest, as mentioned earlier, the quantum of energy $h v_{0}$ corresponding to the rest mass is vibrating as a standing wave packet with a very significantly high frequency. The wave function of the packet at rest in its reference frame is given by the equation (10). Because of the substantially high frequency of the standing wave packet, when it starts to move even with a small velocity, special relativistic effects become manifest in its reference frame $S^{\prime}$ as observed from the laboratory frame $S$. This is a concept brought forward from de Broglie's original thoughts by some recent authors in their treatment of the de Broglie wave [24, 25]. A cogent presentation has been advanced by Shanahan [24]. However, he had to propose a model particle with a standing wave packet at rest. In this presentation the wave packet is revealed to be a natural feature from its very origin.

With a boost velocity $v$ in the $x$ direction and applying the Lorentz transformations

$$
\begin{aligned}
x^{\prime} & =\gamma(x-v t), \\
t^{\prime} & =\gamma\left(t-\frac{v x}{c^{2}}\right),
\end{aligned}
$$

where $\gamma$ is the Lorentz factor

$$
\gamma=\frac{1}{\sqrt{1-\frac{v^{2}}{c^{2}}}}
$$

equation (10) for the standing wave-packet becomes [24]

$$
\begin{aligned}
\psi(x, t) & =W(\gamma(x-v t)) e^{-l m_{0} c^{2} \gamma\left(-\frac{v x}{c^{2}}+t\right) / \hbar} \\
& =W(\gamma(x-v t)) e^{\left(l \gamma m_{0} v x-l \gamma m_{0} c^{2} t\right) / \hbar} \\
& =W(\gamma(x-v t)) e^{(l p x / \hbar)-(l E t / \hbar)}
\end{aligned}
$$

Comparing equation (14) with that of a transverse wave, we can easily identify the wave number $\kappa=p / \hbar$ as the postulated de Broglie wave number with the wave length $\lambda=h / p$. Equation (14) shows that the standing wave packet is now a Lorentz shifted wave packet moving with velocity $v$ and whose space phase is modulated by the complex quantity that involves the momentum $p=m v$.

From the above investigation, it is clear that the wellknown de Broglie wave length $\lambda$ associated with a particle is not really an independent wave but is seen as such due to relativistic effects producing a phase modulation of the wave packet that caries the energy.

\section{Group velocity of the wave packet}

The following analyses ascertain that the group velocity of the wave packet is the translational velocity $v$. Because of the involvement of the velocity $v$, it is more convenient for this purpose to use the Einstein's energy and momentum relations rather than the equivalent Planck formulae

$$
\begin{aligned}
E & =\gamma m_{0} c^{2} \\
p & =\gamma m_{0} v .
\end{aligned}
$$

Since $p=\hbar \kappa$, for the group velocity we have

$$
\begin{aligned}
v_{g} & =\frac{\partial \omega}{\partial \kappa}=\frac{\partial E}{\partial p}=\frac{\partial E}{\partial v}\left(\frac{\partial p}{\partial v}\right)^{-1} \\
& =\frac{\partial}{\partial v} \frac{m_{0} c^{2}}{\sqrt{1-\frac{v^{2}}{c^{2}}}}\left(\frac{\partial}{\partial v} \frac{m_{0} v}{\sqrt{1-\frac{v^{2}}{c^{2}}}}\right)^{-1}
\end{aligned}
$$

Using the quotient and chain rules of differentiation,

$$
v_{g}=\frac{m_{0} v}{\left(1-\frac{v^{2}}{c^{2}}\right)^{\frac{3}{2}}}\left(\frac{m_{0}}{\left(1-\frac{v^{2}}{c^{2}}\right)^{\frac{3}{2}}}\right)^{-1}=v
$$

with $v$ being the velocity of a particle. (Since the phase velocity of the de Broglie wave is $\omega / \kappa=c^{2} / v$, it cannot be involved in the transport of energy at superluminal velocity.) From equation (14), the wave-packet moving with the group velocity $v$ and representing the particle of velocity $v$ is described by the familiar wave function:

$$
\psi(x, t)=W(\gamma(x-v t)) e^{l(\kappa x-\omega t)}
$$

where $\kappa=p / \hbar$ and $\omega=E / \hbar=2 \pi \gamma v_{0}$. In the argument of the exponent, it is necessary to use the de Broglie phase modulation wave number $\kappa$ for a massive particle like an electron [24], whereas for a massless particle such as a photon, the wave number $k$ of the wave packet is used, which appears in equation (3). 


\section{Kinematics of a quantum particle}

Since a particle like an electron in motion is represented by a wave function as given by the equation (20), its kinematics cannot be described by the classical equations of motion. Instead, it requires the use of an equation like the Schrödinger equation, which for a non-relativistic particle is given by

$$
\imath \hbar \frac{\partial}{\partial t} \psi(x, t)=\left[-\frac{\hbar^{2}}{2 m} \nabla^{2}+V(x)\right] \psi(x, t)
$$

where $V(x)$ is the classical potential and the wave function $\psi(x, t)$ is normalized, since the sum total of all the probabilities has to be always one:

$$
\int_{-\infty}^{+\infty} \psi^{*}(x, t) \psi(x, t) d x=1 .
$$

The wave function evolves impeccably in a unitary way. However, when the particle inevitably interacts with a classical device like a measuring apparatus, the wave function undergoes a sudden discontinuous change known as the wave function collapse. Although it is an essential postulate of the Copenhagen interpretation of quantum mechanics, the phenomenon has long been perplexing to the physicists [26, p. 786]. However, a behavior like this would be a natural consequence of the distinctive nature of a quantum particle described in this work. In a measurement, since the holistic wave packet only in its totality always contains the conserved quantities of a particle like an electron such as its energymomentum, charge, spin and so on, it must be taken all at once or not at all. In other words, for measurement, the collapse of the wave function is essential since the entire wave packet holistically representing the particle has to be appropriated.

Parts of the wave function that might spread to a considerably large distance can also terminate instantaneously by the process involved in a credible quantum mechanical Einstein-Rosen bridge [20] and experimentally demonstrated in quantum entanglement of a single photon [27]. The collapse of the entire wave packet in one place then prevents its appearance in any other place.

Thus, the very weave of our universe appears to support the objective reality of the wave function, which represents a natural phenomenon and not just a mathematical construct. Furthermore, the nature of reality of the wave function described in this work indeed requires the observed collapse of the wave function. This is offered as a proof of the ontology of the wave function. Other confounding properties of a quantum particle also follow from the nature of the wave described here.

As elaborated before [11], the renowned uncertainty principle is an inherent property of any wave packet. Due to dispersion, the wave packet would spread out rather quickly. And because the particle is actually a (holistic) wave packet only the probability of the detection of its particle characteristic in a measurement can be predicted. This is consistent with the customary assumption that the wave function is a function of probability amplitudes. Also, since the wave packet is spread out, before detection, the particle has the probability of being observed at more than one place. Therefore, before detection, the particle would appear to be present simultaneously at different places at the same time.

\section{Conclusions}

By the arguments presented in this work, it should be reasonably evident that the wave-or more particularly the wave packet - associated with a material particle in the atomic dimensions is not just a fictitious mathematical vehicle for predicting results by solving the algorithm of quantum mechanics. It represents an objective reality, although not quite a classical one because of the inherently wavelike nature of the particle.

The principal aspect to bear in mind is that only the sum total of all the irregular disturbances in the quantum fields that comprise the travelling wave packet at any instant adds up to the mass, energy-momentum, charge and other conserved quantities of the particle. Consequently, it has to be taken all at the same time or not at all.

By this measure, the enigma of wave-particle duality is deciphered. Likewise, other apparently bizarre quantum behaviors such as the simultaneous existence of a quantum particle in more than one place, the uncertainty principle, achieving only the prediction of the probability rather than actuality of finding a particle in an experiment, the Born rule, and wave function collapse can be given a satisfactory explanation thereby mitigating the perception of quantum weirdness that is so confounding to scientists and even more so to the general public. Now, nearly a century after the formulation of quantum mechanics, it is incumbent upon science to dispel the perception that the quantum core of our daily reality is of questionable realism.

\section{Acknowledgements}

The author wishes to thank Zvi Bern and James Ralston for valuable discussions. 


\section{References}

[1] Schilpp PA. Albert Einstein: Philosopher-Scientist. Library of Living Philosophers, Volume 7, Evanston, Illinois: Open Court Publishing Company, 1949.

[2] Isaacson W. Einstein: His Life and Universe. New York: Simon \& Schuster, 2008.

[3] Leifer MS. Is the quantum state real? An extended review of $\psi$-ontology theorems. Quanta 2014; 3(1): 67-155. doi:10.12743/quanta.v3i1.22

[4] Colbeck R, Renner R. Is a system's wave function in one-to-one correspondence with its elements of reality? Physical Review Letters 2012; 108(15): 150402. arXiv:1111.6597, doi:10. 1103/PhysRevLett. 108.150402

[5] Pusey MF, Barrett J, Rudolph T. On the reality of the quantum state. Nature Physics 2012; 8(6): 475-478. arXiv: 1111.3328, doi:10.1038/nphys2309

[6] Einstein A. Über einen die Erzeugung und Verwandlung des Lichtes betreffenden heuristischen Gesichtspunkt. Annalen der Physik 1905; 17(6): 132-148. doi : 10.1002/andp.19053220607

[7] Einstein A. On a heuristic point of view about the creation and conversion of light. In: The Collected Papers of Albert Einstein. Volume 2: The Swiss Years: Writings, 1900-1909 (English translation supplement). Beck A (translator), Princeton: Princeton University Press, 1905, pp. 86-103. http://einsteinpapers.press. princeton.edu/vol2-trans/100

[8] Davisson CJ, Germer LH. Reflection of electrons by a crystal of nickel. Proceedings of the National Academy of Sciences 1928; 14(4): 317-322. doi: 10.1073/pnas.14.4.317

[9] Thomson GP, Reid A. Diffraction of cathode rays by a thin film. Nature 1927; 119(3007): 890. doi : $10.1038 / 119890 \mathrm{a0}$

[10] W. Moore, Schrödinger: Life and Thought. Cambridge: Cambridge University Press, 1989.

[11] Bhaumik ML. Was Albert Einstein Wrong on Quantum Physics? Quanta 2015; 4(1): 35-42. doi: 10.12743/quanta.v4i1.47

[12] Weinberg S. The Quantum Theory of Fields, Volume 1: Introduction. Cambridge: Cambridge University Press, 1995.
[13] Wilczek FA. The Lightness of Being: Mass, Ether, and the Unification of Forces. New York: Basic Books, 2008.

[14] Susskind L. Particle Physics: 1. Basic Concepts. Stanford Continuing Studies, 2009. https://www.youtube.com/view_play_ list? $p=768 \mathrm{E} 1383 \mathrm{EA79C603}$

[15] Kuhlmann M. Quantum field theory. In: Stanford Encyclopedia of Philosophy, Zalta EN, Nodelman U, Allen C (editors), Stanford: Stanford University, 2012. http://plato.stanford.edu/entries/ quantum-field-theory/

[16] Srednicki M. Quantum Field Theory. Cambridge: Cambridge University Press, 2006. http://web. physics.ucsb.edu/ mark/qft.html

[17] Zee A. Quantum Field Theory in a Nutshell. Princeton: Princeton University Press, 2003.

[18] Wilczek FA. Fantastic Realities: 49 Mind Journeys And a Trip to Stockholm. Singapore: World Scientific, 2006. doi : 10.1142/6019

[19] Brodsky SJ, Franke VA, Hiller JR, McCartor G, Paston SA, Prokhvatilov EV. A nonperturbative calculation of the electron's magnetic moment. $\mathrm{Nu}$ clear Physics B 2004; 703(1-2): 333-362. arXiv: hep-ph/0406325, doi:10.1016/j.nuclphysb. 2004.10 .027

[20] Bhaumik ML. Reality of the wave function and quantum entanglement. 2014; arXiv: 1402.4764

[21] Lederman LM. The God Particle: If the Universe Is the Answer, What Is the Question?. Boston: Houghton Mifflin Company, 2006.

[22] Einstein A. Ist die Trägheit eines Körpers von seinem Energieinhalt abhängig? Annalen der Physik 1905; 18(13): 639-641. doi:10.1002/ andp.19053231314

[23] Einstein A. Does the inertia of a body depend upon its energy content? In: The Collected Papers of Albert Einstein. Volume 2: The Swiss Years: Writings, 1900-1909 (English translation supplement). Beck A (translator), Princeton: Princeton University Press, 1905, pp. 172-174. http://einsteinpapers.press. princeton.edu/vol2-trans/186

[24] Shanahan D. The de Broglie wave as evidence of a deeper wave structure. 2015; arXiv: 1503.02534 
[25] Logiurato F. Relativistic derivations of de Broglie and Planck-Einstein equations. 2012; arXiv: 1208. Q119

[26] Penrose R. The Road to Reality: A Complete Guide to the Laws of the Universe. London: Jonathan Cape, 2004.
[27] Fuwa M, Takeda S, Zwierz M, Wiseman HM, Furusawa A. Experimental proof of nonlocal wavefunction collapse for a single particle using homodyne measurements. Nature Communications 2015; 6: 6665. arXiv:1412.7790, doi:10. $1038 /$ ncomms 7665 